Agnès Lorrain

\title{
Des prologues bibliques d'origine chrysostomienne : Les Arguments attribués à Théodoret et à Théophylacte sur les épîtres pauliniennes
}

DOI 10.1515/zac-2015-0037

\begin{abstract}
This study shows that the argumenta to the Pauline letters attributed to Theodoret in the catena of Oecumenius are without doubt summaries of John Chrysostom's argumenta to these letters, and that they relate to those handed down in the catena of Theophylact. First, we will draw up a list of testimonies of the summaries attributed to Theodoret-manuscripts of catenae and Pauline epistles-and state the hypothesis of a link with the transmission of Oecumenius' catena. After proving the dependence of John Chrysostom and the link with Theophylact, we will bring forward several hypotheses about the precise relations between the three series of argumenta. We will underline the importance of these texts as testimonies of the transmission and reception of John Chrysostom's text, as well as of the technique of summary in Late Antiquity, and we will indicate some directions for further research.
\end{abstract}

Keywords : John Chrysostom; Oecumenius; Euthalius; paratext; summarization technique; biblical manuscripts; exegetical catena; prologue; Theodoret of Cyrus; Theopylact of Ohrid

À Mme le Pr. Marie-Odile Boulnois, en témoignage de ma reconnaissance

Les Arguments d'Euthalius sur les épîtres pauliniennes ont fait l'objet de plusieurs travaux récents, dans le cadre d'études sur l'apparat d'Euthalius. ${ }^{1}$ Cependant,

1 Cf. Vemund Blomkvist, trad., Euthalian Traditions : Text, Translation and Commentary (TU 170 ; Berlin, 2012) ; Louis C. Willard, A Critical Study of the Euthalian Apparatus (Arbeiten zur neutestamentlichen Textforschung 41; Berlin, 2009); Nils A. Dahl, «The «Euthalian Apparatus ` and the Affiliated «Argumenta, > 》 dans Studies in Ephesians : Introductory Questions, Text- \& EditionCritical Issues, Interpretation of Texts and Themes (éd. David Hellholm ; WUNT 131; Tübingen,

Agnès Lorrain : Université Paris-Sorbonne - Antiquité classique et tardive, 28 rue Serpente, Paris 75006, France / Universität Basel - ParaTexBib-Projekt, Nadelberg 10, Basel 4051, Schweiz, e-Mail : alorrain217@gmail.com 
les Arguments attribués à Théodoret, que l'on y trouve adjoints dans quelques témoins, ne semblent pas avoir retenu l'attention. ${ }^{2}$ Nous avons été conduite à nous y intéresser en cherchant à rassembler la tradition manuscrite de l'In epistulas Pauli de Théodoret. ${ }^{3}$ En effet, certains recueils d'épîtres pauliniennes ainsi que des chaînes comportent des Arguments attribués à cet auteur, qui ne correspondent cependant pas à ceux que l'on trouve dans son commentaire de l'Apôtre, et ne présentent même pas de ressemblances avec ceux-ci : nous nous sommes demandé s'ils pouvaient être du même auteur. En outre, plusieurs manuscrits de chaînes contenant ces Arguments ont été considérés par erreur comme des témoins directs du commentaire de l'évêque de Cyr. Pour le Vatopedinus 239, l'erreur du catalogue reproduit celle de la reliure, qui reprend le titre inscrit par

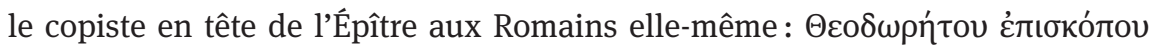

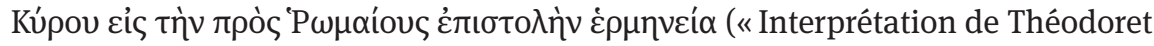
évêque de Cyr sur l'Épître aux Romains »). ${ }^{4}$ Cette confusion nous a amenée à nous interroger sur la paternité, les sources et la date de ces Arguments. C'est ce dont nous voudrions rendre compte dans les lignes qui suivent.

2000), 231-275. Les Arguments d'Euthalius se trouvent notamment dans l'Editio epistolarum Pauli (PG 85:748-788, passim) qui lui est attribuée, reprise et traduite en anglais par Blomkvist, trad., Euthalian Traditions (voir ci-dessus), et avec la chaîne d'Ecumenius (PG 118-119, passim). 2 Ces Arguments sont édités avec la chaîne d'Ecumenius, sous le titre Theodoreti (PG 118-119, passim). Voir aussi Hermann von Soden, Die Schriften des Neuen Testaments in ihrer ältesten erreichbaren Textgestalt : hergestellt auf Grund ihrer Textgeschichte 1,1: Untersuchungen (Berlin, 1902), 350-354. Nous donnons dans l'annexe 1 (pages 494-496) une liste des manuscrits que nous avons étudiés et qui sont mentionnés dans ces pages, avec leur bibliographie sommaire. 3 Cf. Théodoret, Interpretatio in epistulas Pauli (PG 82:36-877). Nous préparons actuellement, dans le cadre d'une thèse de doctorat, l'édition critique et la traduction française du Prologue et de l'In Romanos.

4 Vatopedinus 239, f. 6v (nous normalisons l'orthographe). Erreur d'autant plus étonnante qu'elle apparaît à un endroit où il n'y a pas d'Argument attribué à Théodoret, comme nous le verrons. Paul M. Parvis, Theodoret's Commentary on the Epistles of St Paul : Historical Setting and Exegetical Pratice (thèse de doctorat ; Oxford, 1975), 9-10, avait repéré l'erreur sur ce manuscrit. Les erreurs sur le Coislinianus 27, originaire de la Grande Laure du Mont Athos, ainsi que sur le Chisianus R. VIII 55, avaient déjà été constatées par Karl Staab, Pauluskatenen: Nach den handschriftlichen Quellen untersucht (Scripta Pontificii Instituti Biblici 4; Rome, 1926), 145-146, 103-104. Il faut

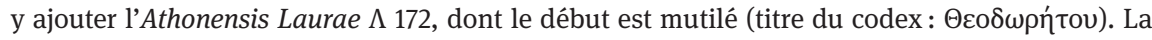
parenté entre ces manuscrits serait à étudier de plus près. 


\section{Première enquête dans la tradition manuscrite}

L'examen des manuscrits, à commencer par ceux répertoriés comme contenant le commentaire ou les Arguments de Théodoret, permet de dresser une première liste et de proposer quelques pistes de réflexion concernant cette série d'Arguments, dont plusieurs témoins remontent $\mathrm{au} \mathrm{x}^{\mathrm{e}}$ siècle. ${ }^{5}$

D'abord, on constate une diversité importante du nombre et du choix des éléments de cette série. Toutes les épîtres sont concernées, à l'exception de Romains et 1 Thessaloniciens, pour les raisons que l'on verra. Toutefois, plusieurs manuscrits contiennent seulement certains de ces Arguments, par exemple sur 2 Corinthiens et Éphésiens, ou sur Philippiens, Colossiens, Tite, sans que l'on puisse regrouper les témoins sur ce critère. ${ }^{6}$

Nous avons trouvé ces Arguments dans deux types de codices, d'une part dans un petit nombre de recueils d'épîtres pauliniennes, d'autre part dans des manuscrits transmettant diverses formes de la chaîne d'Ecumenius, et même dans certains de ses plus anciens témoins. ${ }^{7}$ Ils se situent la plupart du temps après l'Argument d'Euthalius, qui est lui-même beaucoup plus fréquent, et sont parfois suivis d'un troisième. Seuls l'Atheniensis 100 et le Vaticanus gr. 1430 contiennent la série qui nous intéresse sans autres Arguments, mais ce sont aussi des représentants de la chaîne d'Ecumenius. Il faut ajouter que, dans le Sinaiticus gr. 274, les Arguments, à l'exception de celui sur 2 Corinthiens qui suit celui d'Euthalius et précède le texte biblique sans chaîne, sont rassemblés en un recueil à la fin du codex.

5 L’annexe 2 (pages 496-497) fournit deux tableaux précisant quels Arguments contient chaque témoin, et sous quelle forme. Nous avons consulté des microfilms de l'Institut für neutestamentliche Textforschung de Münster, et certains manuscrits de la Bibliothèque Vaticane et de la Bibliothèque Marcienne de Venise, en commençant par les manuscrits les plus anciens.

6 Nous n'avons pas pu expliquer l'absence de tel Argument par des causes matérielles, comme le manque de place en fin de page, mais ce type de facteur a pu intervenir au niveau des modèles des témoins concernés.

7 Les principales formes de la chaîne sont représentées, à savoir, selon la classification de Staab, Pauluskatenen (voir note 4), 99-183: «Normaltypus» (Palatinus gr. 10, Marcianus gr. Z. 34), « erweiterter Typus» (Coislinianus 27, Parisinus gr. 219, Marcianus gr. Z. 33 et Marcianus gr. Z. 546), «Spezialtypus» (Vaticanus gr. 1430). Sur le « sekundärer Erweiterungs-Typus, » voir les manuscrits mentionnés à la note 26 . 
Dans tous les manuscrits, ces pièces sont, au moins pour certaines, attribuées à Théodoret. ${ }^{8}$ Nous n'avons trouvé aucun de ces Arguments attribué à un autre auteur. Il n'est donc pas possible de dater l'attribution. Cependant, quelques détails permettent de supposer un lien avec la transmission de la chaîne d'Ecumenius. D’abord, en ce qui concerne les recueils sans chaîne, l'ordre des épîtres correspond à celui que l'on observe dans cette chaîne, et qui n'est pas l'ordre le plus représenté en-dehors de celle-ci. ${ }^{9}$ De plus, dans les mêmes manuscrits, ces Arguments suivent ceux d'Euthalius, comme c'est le cas dans la chaîne. En ce qui concerne les manuscrits avec chaînes, certains Arguments sont disposés, en particulier dans des témoins très anciens, comme le serait un premier extrait de chaîne, quoiqu'il ne soit pas compté dans la numérotation des scholies. En revanche, dans ces mêmes manuscrits, la mise en page de l'Argument d'Euthalius distingue toujours celui-ci de la chaîne, soit qu'il soit écrit en pleine page alors que la chaîne a une disposition marginale, soit qu'un titre, éventuellement précédé d'une frise, marque le début de la chaîne. Dans ces cas, la mise en page elle-même fait de ces Arguments attribués à Théodoret un élément de la chaîne d'Eccumenius. ${ }^{10}$

\section{Paternité et sources}

Ces Arguments ne présentant pas de point commun avec ceux que Théodoret place dans l'In epistulas Pauli avant le commentaire de chacune des épîtres, il reste à se demander s'ils peuvent cependant être du même auteur, comme le veut la tradition manuscrite, ou s'il faut les attribuer à un autre. Or, l'enquête sur les sources conduit sans le moindre doute au résultat suivant: ces textes sont de simples résumés des introductions de Jean Chrysostome sur les épîtres

8 On trouve parfois l'abréviation $\Theta \varepsilon$ co ., usuelle dans les chaînes aussi bien pour Théodore que pour Théodoret, mais jamais le nom Théodore en entier. Dans l'Ambrosianus Z 34 sup., les Arguments sur Philippiens et Colossiens ne sont pas attribués. Quant aux Arguments d'Euthalius, ils apparaissent sans nom d'auteur dans les manuscrits consultés.

9 Il s'agit de l'ordre devenu canonique. Par ailleurs, la majorité des manuscrits en général semblent avoir Hébreux après 2 Thessaloniciens. Cf. David Trobisch, Die Entstehung der Paulusbriefsammlung : Studien zu den Anfängen christlicher Publizistik (Novum Testamentum et Orbis Antiquus 10 ; Fribourg [Suisse], 1989), 12-62. C'est du reste le cas de la chaîne Parisinus gr. 219. 10 Sur la question de la disposition originelle de ces Arguments, voir la discussion ci-dessous (p. 490-491). 
correspondantes. ${ }^{11}$ En outre, ils présentent des similarités importantes avec les Arguments édités avec le commentaire de Théophylacte d'Achrida (1050-1108). ${ }^{12}$ Les Arguments attribués à Théodoret étant plus courts que ceux attribués à Théophylacte, nous appelons désormais les premiers « résumés courts » et les seconds, « résumés longs.»

D'un point de vue macroscopique, il faut noter que l'absence d'Argument sur 1 Thessaloniciens concerne les trois corpus. L'absence de résumé court sur Romains ne s'explique pas aussi facilement, mais pourrait être liée au fait qu'une grande partie de l'Argument de Jean Chrysostome sur cette épître est en réalité une introduction à l'ensemble des épîtres pauliniennes. À l'échelle de chaque épître, les textes attribués à Théophylacte et à Théodoret sont des résumés de passages sélectionnés dans l'Argument de Jean Chrysostome: mêmes questionnements et mêmes idées, ordonnées de la même façon. La dépendance des deux séries d'Arguments par rapport à ceux de Jean Chrysostome est tout à fait certaine. On pourrait en fournir la preuve en mettant, pour chaque épître, les trois textes en regard. On donnera simplement en exemple le cas le plus évident, celui des Arguments sur Philémon:

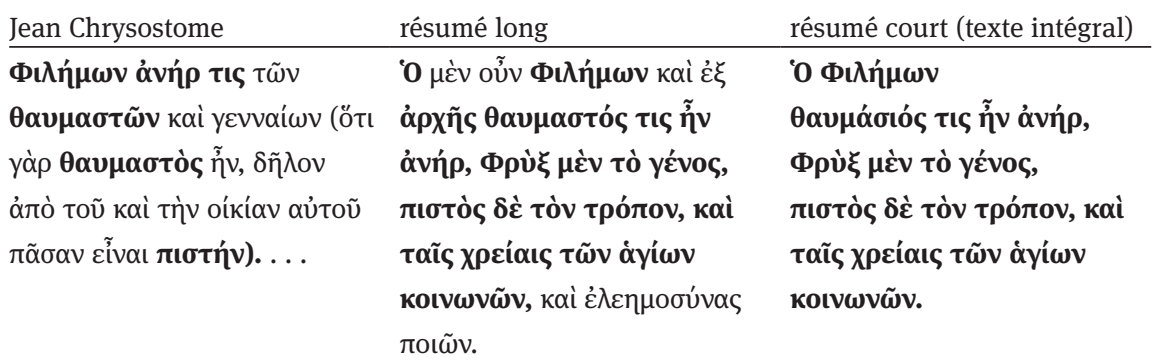

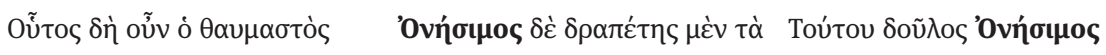

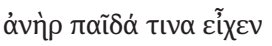
$\pi \rho \tilde{\omega} \tau \alpha$.

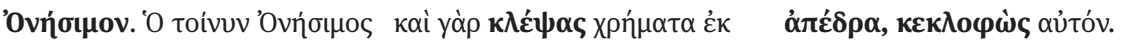

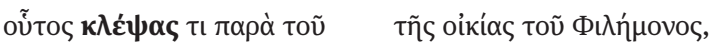

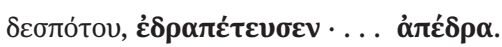

11 Cf. Jean Chrysostome, Commentaria in epistulas Pauli (PG 60-63, passim). Ces textes précédant chaque fois l'interprétation de la première péricope sont présentés, suivant les cas, comme un Argument séparé (Romains, 1 Corinthiens, Éphésiens, Philippiens, 1 Timothée, Philémon, Hébreux), comme une homélie liminaire (2 Thessaloniciens), ou comme le début de la première homélie (2 Corinthiens, Colossiens, 2 Timothée, Tite) ou de la première partie du commentaire (Galates). Pour ne pas alourdir le texte, nous parlerons d'Arguments sans distinction.

12 Cf. Théophylacte de Bulgarie, Commentarii in Pauli epistulas (PG 124-125, passim). 


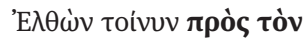

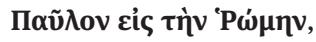

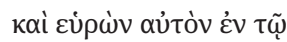

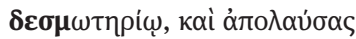

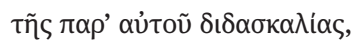

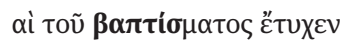

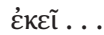

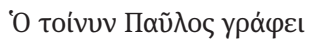

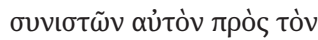

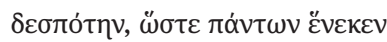

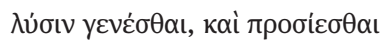

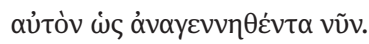

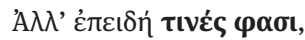

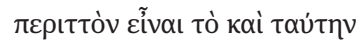

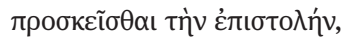

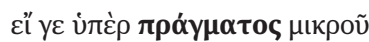

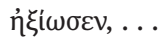

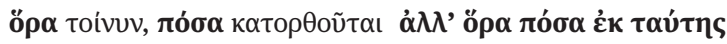

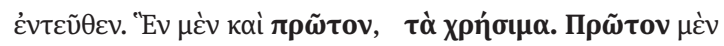

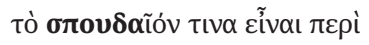

$\pi \alpha \dot{v} v \tau \alpha$.

$\Delta \varepsilon v \dot{\tau \varepsilon \rho o v, ~ o ̈ \tau ı ~ \tau o ̀ ~ \delta o v \lambda เ \kappa o ̀ v ~}$

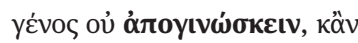

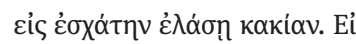

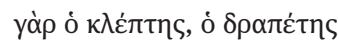

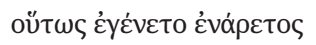

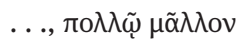

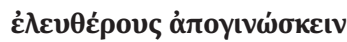

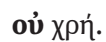

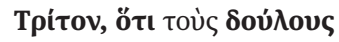

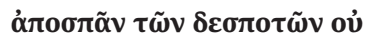

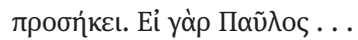

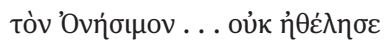

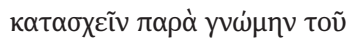

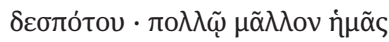

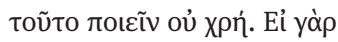

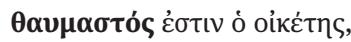

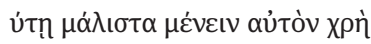

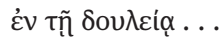

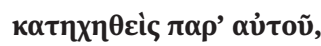

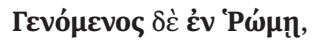

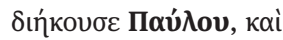

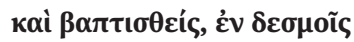

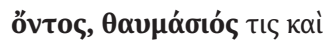

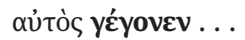

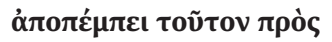

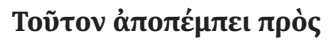

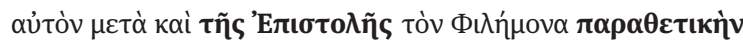

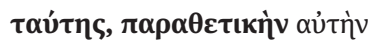

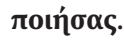

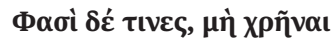

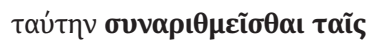

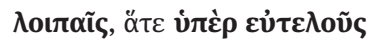

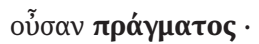

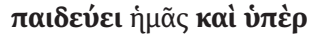

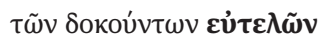

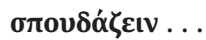

$\Delta \varepsilon v \dot{\tau \varepsilon \rho o v ~ \delta \varepsilon ́, ~ o ̈ \tau เ ~ \varepsilon i ́ ~}$

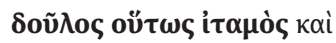

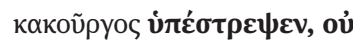

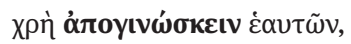

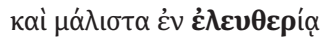

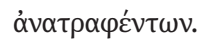

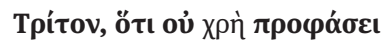

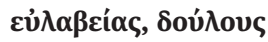

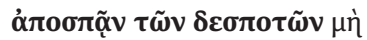

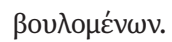

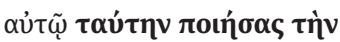

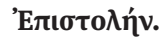

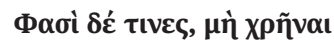

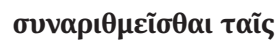

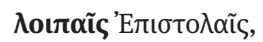

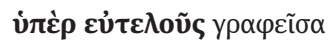

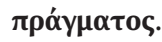

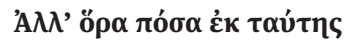

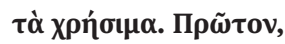

$\pi \alpha ı \delta \varepsilon v ́ \varepsilon เ ~ \kappa \alpha i ̀ ~ v ่ \pi \varepsilon ̀ \rho ~ \varepsilon v ่ \tau \varepsilon \lambda \tilde{\omega} v$

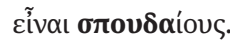

$\Delta \varepsilon \dot{\tau \varepsilon \rho o v, ~ o ̈ \tau เ ~ \varepsilon i ~ \delta o v ̃ \lambda o \varsigma ~}$

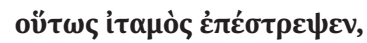

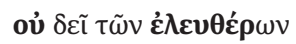

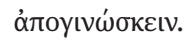

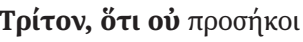

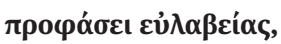

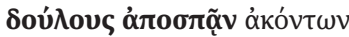
$\tau \tilde{\omega} \boldsymbol{\nu} \delta \varepsilon \sigma \pi 0 \tau \tilde{\omega} v$. 


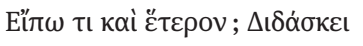

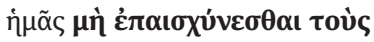

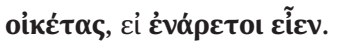

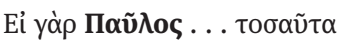

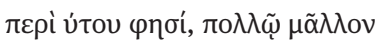

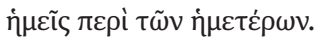

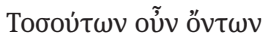

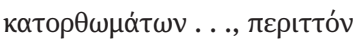

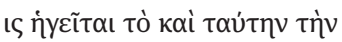

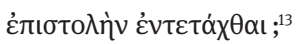

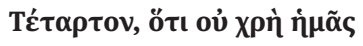

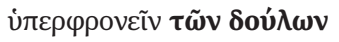

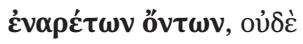

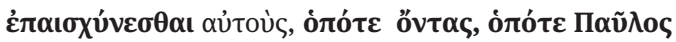

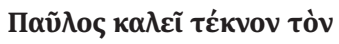
'Ovท́бıนоv.

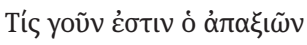

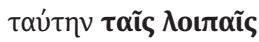

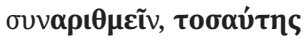

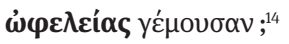

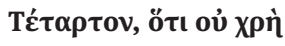

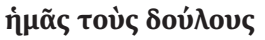

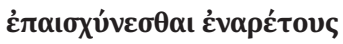

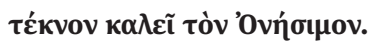

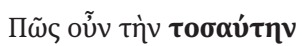

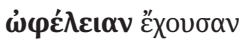

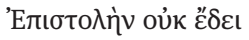

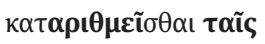

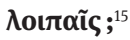

Les trois textes présentent les mêmes idées dans le même ordre, sans qu'il s'agisse d'une paraphrase du texte de l'épître. Le contexte est d'abord évoqué : qualité de Philémon ( $\theta \alpha u \mu \alpha \sigma \tau o ́ s, ~ \theta \alpha u \mu \alpha ́$ бı vol ( $\kappa \lambda \varepsilon ́ \pi \tau \varepsilon ı v)$ commis par son esclave Onésime, fuite de celui-ci, sa rencontre avec Paul en prison à Rome, sa conversion, son baptême. Ensuite, les Arguments énoncent quatre « utilités » $(\chi \rho \eta ́ \sigma \iota \mu \alpha)$ de l'épître : celle-ci enseigne premièrement

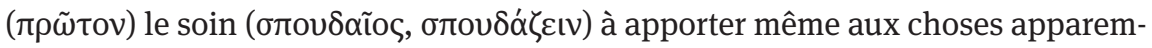
ment sans importance, apprend deuxièmement ( $\delta \varepsilon u ́ \tau \varepsilon \rho o v)$ à ne pas désespérer

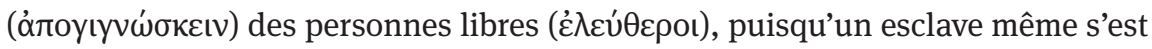

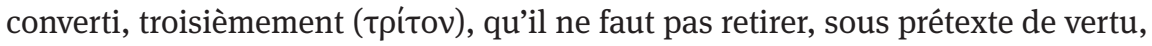

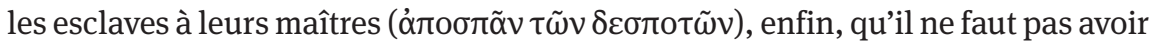

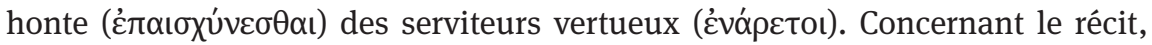

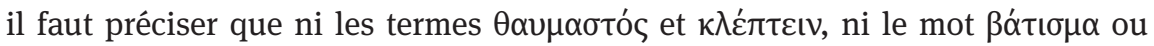
$\beta \alpha \pi \tau i \zeta \zeta c ı$, ni l'idée précise de fuite ne se trouvent dans l'épître. Quant aux parties de l'Argument chrysostomien laissées de côté, outre bien des détails à l'intérieur des phrases - évoquant par exemple la vertu de Paul - elles sont nombreuses : citations de l'épître fondant les différents éléments du récit, long développement sur l'utilité de rapporter les moindres faits et gestes des apôtres, ou encore sur l'avantage de rester dans l'état d'esclave.

Les éléments permettant de supposer la parenté entre les résumés sur Philémon sont de deux types. Du point de vue du contenu, un détail commun est absent

13 Jean Chrysostome, In epistulam ad Philemonem commentarius, argumentum (PG 62:701-704). Voir notre traduction des trois textes dans l'annexe 3, p. 498-499.

14 Théophylacte de Bulgarie, Expositio Epistulae divi Pauli ad Philemonem (PG 125:172-173). 15 Ps.-Théodoret, Argumentum epistulae ad Philemonem Pauli Apostoli (PG 119:264). 
chez Jean Chrysostome, l'ajout de l'origine phrygienne du destinataire. ${ }^{16}$ Toutefois, cela peut relever de la variante textuelle dans l'œuvre de Jean Chrysostome. Du reste, nous avons trouvé la même mention dans une autre forme de résumé de l'Argument. ${ }^{17}$ D’autre part, on peut observer un réagencement par rapport au texte de Jean Chrysostome : l'attribution à l'esclave de l'adjectif $\theta \alpha u \mu \alpha ́$ óı lisé pour le maître est mise en valeur par sa proximité avec la première utilisation du qualificatif, alors qu'elle se trouve indirectement suggérée dans l'Argument de Jean Chrysostome dans la troisième utilité de l'épître. ${ }^{18}$ Bien plus convainquantes encore sont les nombreuses ressemblances littérales entre les deux résumés : très souvent, ceux-ci sont plus proches l'un de l'autre que chacun ne l'est de Jean Chrysostome. Beaucoup de phrases sont identiques à quelques mots près et les seules différences affectant le sens sont des détails absents du résumé court, qui, par exemple, évite la redondance à propos de la fuite, omet la précision sur les objets volés ainsi que celle sur l'écoute de Paul, utilise seulement le premier

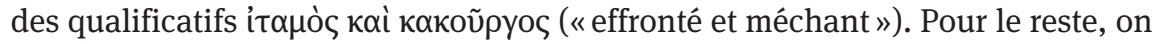

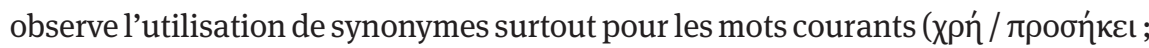

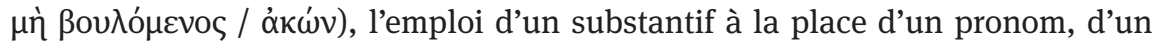

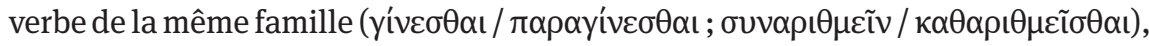
ou encore une formulation différente de la question finale. Un certain nombre de ces détails peuvent relever de la simple variante textuelle.

Par ailleurs, pour revenir à l'ensemble de ces résumés, certaines différences entre les deux séries indiquent un rapport complexe entre les deux traditions. On peut d'abord affirmer que les résumés longs ne peuvent avoir pour seule source les résumés courts, car ils empruntent à Jean Chrysostome des développements

16 Dans les Arguments sur les autres épîtres, on trouve les détails suivants : ajout du nom de Néron dans les résumés sur 2 Timothée (Théophylacte de Bulgarie, Expositio Epistulae divi Pauli ad II Timotheum [PG 125:88] ; Ps.-Théodoret, Argumentum epistulae ad II Timotheum Pauli Apostoli

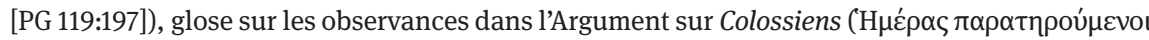

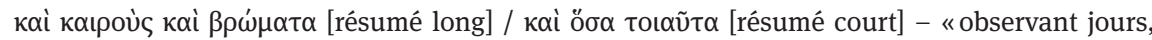
périodes et nourritures» / «et toutes les choses de ce genre»). Cf. Théophylacte de Bulgarie, Expositio Epistulae divi Pauli ad Colossenses (PG 124:1205) ; Ps.-Théodoret, Argumentum epistulae ad Colossenses Pauli Apostoli (PG 119:12).

17 Cet autre résumé, non édité à notre connaissance, se trouve dans le Monacensis gr. 375 et dans le Marcianus gr. Z. 35. Il comprend par ailleurs des éléments issus de Jean Chrysostome et absents des deux résumés étudiés ici. Sur cet Argument et les autres contenus dans ces deux manuscrits, voir note 26.

18 On a observé un seul autre cas de réagencement des idées, à savoir dans les Arguments sur Philippiens: les événements ayant marqué la communauté ont été rassemblés vers la fin des deux résumés, alors que certains d'entre eux étaient évoqués, chez Jean Chrysostome, dans les premières lignes. 
qu'on ne trouve pas dans ceux-ci. Par exemple, à la fin du résumé long sur Tite, la comparaison chrysostomienne entre les reproches adressés par Paul et ceux adressés par le Christ aux pharisiens est citée presque littéralement, ainsi que la réflexion sur la brièveté de la lettre : on ne trouve pas ces éléments dans les résumés courts. Par ailleurs, dans l'unique cas où la dépendance du résumé court par rapport à Jean Chrysostome est moins flagrante - Arguments sur Colossiens ${ }^{19}$ -, on peut, certes, relever des points communs entre les deux résumés - réorganisation des idées pour séparer l'évocation des pratiques et la question de l'origine du Salut, ajout d'exemples sur les observances -, mais la réflexion initiale du résumé court sur le thème de la médiation, non empruntée à Jean Chrysostome, ne se trouve pas dans le résumé long qui, de son côté, offre une synthèse du long développement initial du prédicateur sur la valeur particulière des épîtres de la captivité. ${ }^{20}$

De tels indices dispensent de mentionner en plus certaines ressemblances littérales entre les résumés longs et Jean Chrysostome, contre les résumés courts.

La question de la dépendance des résumés courts par rapport aux résumés longs est délicate, même si on s'attache seulement à l'étude du texte, laissant pour l'instant de côté le problème de chronologie, qui sera évoqué plus loin. En faveur de la dépendance, outre les ressemblances mentionnées plus haut, il faut noter que les développements de Jean Chrysostome sélectionnés pour faire les résumés courts se trouvent toujours dans les résumés longs. On ne peut cependant nier les quelques arguments plaidant pour une autonomie entre les deux séries, même s'ils ne sont pas aussi solides. D’abord, les quelques réflexions des résumés longs qui n'ont pas pour source le texte de Jean Chrysostome tel que nous le connaissons par la $P G$ ne se retrouvent pas dans les résumés courts, ainsi la réflexion préliminaire sur le fait que le Christ n’a rejeté ni les esclaves ni les maîtres (sur Philémon) : ce n'est encore qu'une preuve a silentio et le fait est cohérent avec la longueur relative des deux séries. Concernant certains détails, le résumé court présente parfois le même ordre que l'Argument de Jean Chrysostome, alors que le résumé long s'en écarte, par exemple la mention finale de la priorité

19 Cf. Jean Chrysostome, In epistulam ad Colossenses commentarius, homilia 1,1-2 (PG 62:299-301); Théophylacte de Bulgarie, Expositio Epistulae divi Pauli ad Colossenses (PG 124:1205) ; Ps.Théodoret, Argumentum Epistulae ad Colossenses (PG 119:12). Des extraits sont fournis dans l'annexe 4 (p. 500-501).

20 Le seul écart non encore mentionné entre les résumés courts et les Arguments de Jean Chrysostome est l'ajout d'une citation des Hypotyposeis de Clément d'Alexandrie à la fin de l'Argument sur Hébreux (cf. Ps.-Théodoret, Argumentum epistulae ad Hebraeos Pauli Apostoli (PG 119:276), qui correspond, à quelques variantes près, au fragment transmis par Eusèbe de Césarée, Historia ecclesiastica 6,14,4 (SC 41, 107 Bardy). 
chronologique de Tite sur Timothée est placée au milieu du résumé long. Parmi les ressemblances littérales entre le résumé court et le texte de Jean Chrysostome, on donnera deux exemples parmi les plus probants: la formule interrogative introduisant l'Argument sur 2 Corinthiens, qui disparaît dans le résumé long, et,

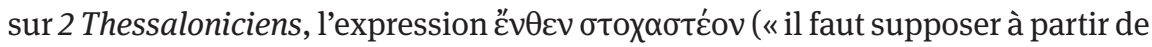

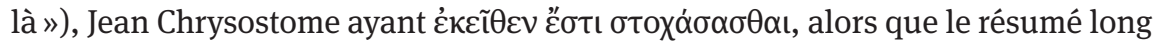
a une formule plus banale, $\delta \tilde{n} \lambda$ ov $\varepsilon \dot{v} \varepsilon \tilde{v} \theta \varepsilon v .{ }^{21}$ Peut-on supposer que les résumés courts que nous avons s'appuient sur un autre état des résumés longs que celui sur lequel nous travaillons ? $^{22}$

Enfin, il faut signaler les nuances entre les différentes épîtres concernant la parenté entre les deux séries de résumés. Si les résumés courts sur Éphésiens, 2 Timothée et Philémon paraissent directement issus des résumés longs sans qu'aucun recours à Jean Chrysostome soit nécessaire, à l'inverse, les rapports sont plus lâches au sujet de 1 Corinthiens, ou encore Hébreux, ce qui toutefois ne met pas en cause la dépendance des résumés courts par rapport à Jean Chrysostome.

Quoi qu'il en soit, la paternité de Théodoret de Cyr sur les résumés courts est peu probable. À notre connaissance, on ne trouve ni chez Théodoret, ni chez ceux qui se sont intéressés à son œuvre, mention de résumés qu'il aurait pu faire de Jean Chrysostome, avant de produire des œuvres exégétiques caractérisées, on le sait, par une utilisation libre de ses sources. Il est beaucoup plus vraisemblable que ces résumés soient l'œuvre d'un scribe quelconque et que l'attribution à Théodoret soit postérieure. Si l'on admet un lien avec la rédaction de la chaîne d'Eccumenius, on peut formuler l'hypothèse suivante : le caténiste ou un éditeur postérieur ${ }^{23}$ aurait placé, comme premier extrait et non à l'extérieur de la chaîne - pour des raisons d'économie de place ou simplement parce que ces introductions étaient considérées comme le début du commentaire ${ }^{24}$-, en tête de chaque épître, un résumé, réalisé ou non par ses soins, de l'Argument de Jean Chrysostome. Constatant ensuite que ces extraits ne correspondaient pas à

21 Cf. Ps.-Théodoret, Argumentum epistulae ad II Thessalonicenses Pauli Apostoli (PG 119:108); Jean Chrysostome, In epistulam ad II Thessalonicenses commentarius, homilia 1,1 (PG 62:469); Théophylacte de Bulgarie, Expositio Epistulae divi Pauli ad II Thessalonicenses (PG 124:1328).

22 Nous envisageons différentes hypothèses en conclusion (p. 492-493).

23 Ces résumés ne sont pas intégrés dans la numérotation des extraits lorsque celle-ci existe. Sur la complexité de la tradition de cette chaîne, cf. Staab, Pauluskatenen (voir note 4), 99-183. 24 Il n'est pas rare, dans d'autres chaînes aussi, que le premier extrait offre une sorte d'introduction sur le livre et non un commentaire du premier verset. Le phénomène se trouve déjà dans les séries d'homélies exégétiques de Jean Chrysostome telles que nous les connaissons (voir note 11), sans que l'on puisse établir de correspondance exacte entre les épîtres concernées dans le cas des chaînes et dans le cas de Jean Chrysostome (voir aussi note 26). 
l'explication du premier verset mais avaient une portée plus générale, certains copistes les auraient séparés de la chaîne proprement dite pour les placer à la suite de l'Argument d'Euthalius. Puis ces résumés auraient circulé dans des manuscrits bibliques sans chaîne. L'hypothèse inverse d'une inclusion postérieure d'éléments d'abord séparés de la chaîne pourrait aussi être envisagée si l'on imagine, à l'origine, une distinction peu claire entre Argument et chaîne. Toutefois, un éventuel passage de la disposition à pleine page à la disposition marginale ne suffirait pas à l'expliquer, puisque des manuscrits anciens à pleine page présentent eux-mêmes une séparation claire entre les Arguments - éventuellement suivis de la liste des capitula - et la chaîne, au moyen d'une frise précédant le titre du livre biblique. ${ }^{25}$

\section{Conclusion}

L'étude des Arguments sur les épîtres pauliniennes attribués à tort à Théodoret offre un témoignage original sur la postérité des œuvres de Jean Chrysostome. Celles-ci n’ont pas seulement été abondamment copiées, entièrement et par extraits, mais aussi résumées de diverses manières, dont les séries évoquées ne sont pas les uniques représentants. ${ }^{26} \mathrm{Il}$ est intéressant de constater que, dans ce

25 Sur l'évolution de la mise en page des chaînes exégétiques, et le rapport avec les genres du commentaire et des scholies dont elles héritent, cf. Gilles Dorival, « Des commentaires de l'Écriture aux chaînes, » dans Le Monde grec ancien et la Bible (éd. Claude Mondésert ; Paris, 1984), 361-386. L'auteur ne se prononce pas sur la mise en page originelle des chaînes sur le Nouveau Testament, dont la rédaction, dit-il, pourrait être postérieure à l'introduction de la disposition marginale.

26 On a trouvé d'autres formes de résumés dans deux manuscrits avec chaîne, Monacensis gr. 375 et Marcianus gr. Z. 35, qui contiennent par ailleurs certains Arguments d'Euthalius mais aucun des «résumés courts » étudiés ici. La présentation de ces Arguments est identique dans les deux témoins et le texte semblable ; l'ordre des épîtres est cependant différent (Hébreux est placé après 2 Thessaloniciens dans le Monacensis gr. 375, à la fin du corpus dans le Marcianus

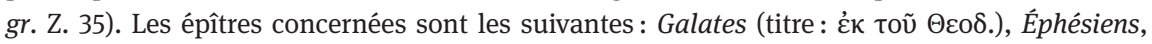
Colossiens, 1 Timothée, 2 Timothée, Tite, Philémon (ces trois derniers commençant avec la formule initiale typique des Arguments d'Euthalius), Hébreux. Dans le Monacensis gr. 375 (non dans le Marcianus gr. Z. 35, mutilé en son début), le premier extrait de la chaîne sur 2 Corinthiens est un résumé du début de la première homélie de Jean Chrysostome, ressemblant au résumé court, mais sans discontinuité entre l'introduction à l'épître et le commentaire du premier verset. Ces textes, dont le degré de ressemblance avec les résumés courts est variable, ne sont pas édités à notre connaissance, sauf celui sur Hébreux (cf. von Soden, Die Schriften des Neuen Testaments [voir note 2], 352-353, variantes de $\alpha$ 209). Leur prise en compte pour mieux comprendre la transmission de ces résumés chrysostomiens est indispensable, comme le montre l'exemple des Arguments sur Philémon évoqué plus haut. 
processus, le nom de Jean Chrysostome a pu être perdu tandis que d'autres y ont été attachés. Celui de Théodoret rejoint une réputation sans doute caricaturale de l'évêque de Cyr : chrysostomien, concis, compilateur.

La question du lien précis entre les deux séries de résumés soulève plus de problèmes que leur rapprochement n'en a résolus. En effet, les ressemblances entre les deux contre le texte édité de Jean Chrysostome excluent l'hypothèse de deux réécritures indépendantes dudit texte. Il faut donc supposer soit un autre ancêtre commun, soit une filiation, soit une situation plus complexe. Envisager un ancêtre commun permettrait d'expliquer les ressemblances avec le texte de Jean Chrysostome de chaque série indépendamment de l'autre. Cela signifierait soit la circulation d'une série de résumés plus ancienne, à laquelle les deux séries considérées auraient repris des formulations, soit l'existence d'une tradition des homélies chrysostomiennes assez différente de celle reflétée par la $P G$. Cette tradition double pourrait avoir existé soit dès l'origine - sachant que les homélies de Jean Chrysostome ont été, au moins en partie, mises par écrit par des tachygraphes -, soit plus tardivement, par des recensions divergentes. Il faudrait alors imaginer des recensions affectant considérablement le style, vu les ressemblances littérales notables entre les deux séries. Et surtout, l'idée d'un ancêtre commun, résumé perdu ou recension différente du texte de Jean Chrysostome, explique mal le fait qu'aucun des développements de Jean Chrysostome sélectionnés pour les résumés courts n'est absent des résumés longs.

L'idée selon laquelle les résumés longs seraient l'unique source des résumés courts semble vraisemblable du point de vue du texte. Les arguments qui s'y opposent sont assez fragiles, et certains indices seraient peut-être rendus invalides par une enquête dans la tradition manuscrite des différentes séries d'Arguments. Toutefois, l'hypothèse pose un problème de chronologie : puisque les résumés courts sont déjà attestés dans des manuscrits du $\mathrm{x}^{\mathrm{e}}$ siècle, sauf à contester la datation de ceux-ci, la source doit être antérieure, ce qui met en cause la paternité de Théophylacte. Est-ce à dire que celui-ci a copié des Arguments existants, ou que ceux-ci ont été ajoutés à son œuvre ? $^{27}$ Peut-on trouver des témoins de ces Arguments dans des manuscrits antérieurs au $\mathrm{xI}^{\mathrm{e}}$ siècle?

Par ailleurs, on peut concevoir que Théophylacte ait utilisé à la fois des résumés existant et le texte de Jean Chrysostome. En effet, si sa dépendance à l'égard de ce dernier est bien connue, son recours à la chaîne d'Ecumenius sur les épîtres pauliniennes a aussi été évoquée par Ernest W. Saunders, selon qui «His com-

27 L'hypothèse ne doit pas être négligée: on trouve un cas analogue à propos de l'édition de son commentaire sur les Actes, dont l'Argument reprend l'apparat d'Euthalius (cf. Blomkvist, Euthalian Traditions [voir note 1], 96-97), aussi transmis avec la chaîne d'Ecumenius (PG 118). 
mentaries represent principally reproductions of Chrysostom, either directly or through the catenae and Oecumenius, though the precise relationships have yet to be defined. ${ }^{28}$ Il pourrait donc avoir utilisé les résumés de Jean Chrysostome trouvés dans la chaîne d'Ecumenius, tout en les complétant à l'aide de la source, puisqu'il avait les deux à sa disposition. Cette hypothèse renvoie à la question de la méthode de Théophylacte, qui demanderait à être creusée par une étude comparative entre son œuvre, celle de Jean Chrysostome et celle d'Ecumenius. ${ }^{29}$

Quelle que soit l'hypothèse envisagée, la nature des ressemblances et des divergences entre les trois séries d’Arguments nécessiterait de travailler sur la base d'éditions critiques, et même de tenir compte d'autres formes de résumés de Jean Chrysostome. En ce qui concerne la tradition manuscrite des résumés courts, l'identification de nouveaux témoins pourrait permettre de préciser le lien avec la chaîne d'Eccumenius et l'origine de l'attribution à Théodoret. Il faudrait approfondir aussi la question de la dépendance ou autonomie de transmission entre ces textes et les Arguments d'Euthalius, voire de la chaîne d'Eccumenius.

Remerciements : Nous présentons ici les premiers résultats d'une réflexion menée en 2012-2013. Une liste beaucoup plus complète des témoins, du moins des manuscrits bibliques, pourra être dressée dans le cadre du projet « ParaTexBib » auquel nous participons, sous la direction des Prs. Martin Wallraff et Patrick Andrist. Je remercie ces derniers, ainsi que mon directeur de thèse, le Pr. Olivier Munnich, pour leur relecture de ces pages et leurs suggestions précieuses. Je souhaite également remercier le Pr. Erich Lamberz, grâce à qui j’ai pu consulter une copie du Vatopedinus 239.

28 Ernest W. Saunders, « Theophylact of Bulgaria as Writer and Biblical Interpreter, » Biblical Research 2 (1957) : (31-44) 44.

29 Une comparaison précise entre Jean Chrysostome et Théophylacte a été réalisée à propos $\mathrm{du}$ commentaire sur les Douze petits prophètes. Cf. Mathilde Aussedat et Matthieu Cassin, « Le prologue du Commentaire sur les petits prophètes de Théophylacte d'Achrida, » Revue des Études Byzantines 68 (2010) : 61-93. Aussedat évoque la «manière [de Théophylacte] de sélectionner, résumer, simplifier, modifier et enrichir ses sources» (ibid., 78). 


\section{Annexe 1: Liste des manuscrits mentionnés ${ }^{30}$}

\subsection{Manuscrits contenant des « résumés courts »}

Atheniensis 100 : Athènes, Ethnikê Bibliothêkê tês Hellados (EBE), 100 (Aland 075), parch. $\mathrm{X}^{\mathrm{e}}$ siècle (parties restaurées : $\mathrm{XV}^{\mathrm{e}}$ siècle), disposition à pleine page. ${ }^{31}$ Vatopedinus 239: Athos, Monê Batopediou, 239 (Aland 2183), parch., XI ${ }^{\mathrm{e}}$ siècle, disposition marginale. ${ }^{32}$

Athonensis Laurae $\Lambda$ 172: Athos, Monê Megistês Lauras, $\Lambda 172$ (Eustratiades 1663, Aland 1772), pap., XIV ${ }^{\mathrm{e}}$ siècle, disposition à pleine page. ${ }^{33}$

Londres, British Museum, Add. 37003 (Aland 2279), parch., XII ${ }^{\mathrm{e}}$ siècle, une colonne. ${ }^{34}$

Ambrosianus E 97 sup.: Milan, Biblioteca Ambrosiana, E 97 sup. (Martini-Bassi 304 ; Aland 614), parch., XII ${ }^{\mathrm{e}}$ siècle, une colonne. ${ }^{35}$

Ambrosianus Z 34 sup. : Milan, Biblioteca Ambrosiana, Z 34 sup. (Martini-Bassi 751; Aland 592), pap., XIII ${ }^{\mathrm{e}}$ siècle (A. D. 1289), une colonne. ${ }^{36}$

30 Pour les manuscrits du Nouveau Testament sans chaîne, nous donnons le nombre de colonnes. Les mentions « disposition marginale » et « disposition à pleine page » concernent les manuscrits de chaîne. Les cotes de catalogues anciens sont indiquées entre parenthèses. Le numéro « Aland » se réfère à Kurt Aland, Kurzgefasste Liste der griechischen Handschriften des Neuen Testaments (Arbeiten zur neutestamentlichen Textforschung 1; Berlin, 1994).

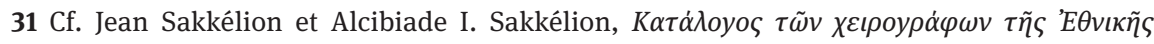

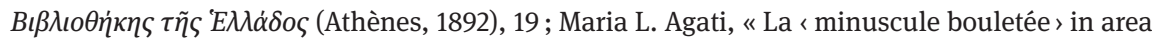
provinciale, » dans Scritture, libri e testi nelle aree provinciali di Bisanzio : Atti del seminario di Erice [18-25 settembre 1988] (éds. Guglielmo Cavallo, Giuseppe de Gregorio et Marilena Maniaci ; Spoleto, 1991), (203-217) 213.

32 Cf. Sophronios Eustratiades et Arcadios, Catalogue of the Greek Manuscripts in the Monastery of Vatopedi on Mt. Athos (Harvard Theological Studies 11; Cambridge [Mass.], 1924), 53 ; Parvis, Theodoret's Commentary (voir note 4), 9-10.

33 Cf. Spyridon et Sophronios Eustratiades, Catalogue of the Greek Manuscripts in the Library of the Laura on Mount Athos with notices from other libraries (Harvard Theological Studies 12; Cambridge [Mass.], 1925), 293.

34 Cf. Marcel Richard, Inventaire des manuscrits grecs du British Museum 1 : Fonds sloane additional, Egerton, Cottonian et Stowe (Paris, 1952), 68. Aland, Kurzgefasste Liste (voir note 30), 178, situe ce manuscrit au xiv ${ }^{\mathrm{e}}$ siècle.

35 Cf. Emidio Martini et Domenico Bassi, Catalogus Codicum Græcorum Bibliothecae Ambrosianae 1 (Milan, 1906), 347-348.

36 Cf. Emidio Martini et Domenico Bassi, Catalogus Codicum Græcorum Bibliothecae Ambrosianae 2 (Milan, 1906), 859-861. 
Moscou, Gosudarstvennyj Istoricheskij Muzej (GIM), Sinod. gr. 098 (Vladimir 094, Aland 1927), olim Hagion Oros, Monê Megistês Lauras, parch., $\mathrm{X}^{\mathrm{e}}$ siècle, disposition marginale. ${ }^{37}$

Coislinianus 27 : Paris, Bibliothèque Nationale de France, Coislin 27 (Aland 1905), parch., $\mathrm{X}^{\mathrm{e}}$ siècle, disposition marginale. ${ }^{38}$

Parisinus gr. 219: Paris, Bibliothèque Nationale de France, gr. 219 (Aland 91), parch., $\mathrm{XI}^{\mathrm{e}}$ siècle, disposition à pleine page. ${ }^{39}$

Sinaiticus gr. 274 : Sinaï, Monê tês Hagias Aikaterinês, gr. 274 (Aland 1244), parch., $\mathrm{x}^{\mathrm{e}}$ siècle, une colonne. ${ }^{40}$

Palatinus gr. 10 : Vatican, Biblioteca Apostolica Vaticana, Palatinus gr. 10 (Aland 1997), parch., $\mathrm{x}^{\mathrm{e}}$ siècle, disposition marginale. ${ }^{41}$

Vaticanus gr. 1430 : Vatican, Biblioteca Apostolica Vaticana, Vaticanus gr. 1430 (Aland 622), parch., $\mathrm{XI}^{\mathrm{e}}$ siècle, disposition à pleine page. ${ }^{42}$

Marcianus gr. Z. 33 : Venise, Biblioteca Nazionale Marciana, gr. Z. 33 (coll. 423; Aland 1923), parch., $\mathrm{x}^{\mathrm{e}} / \mathrm{xI}^{\mathrm{e}}$ siècle, disposition marginale. ${ }^{43}$

37 Cf. Archimandrite Vladimir, Систематическое описание рукописей Московской Синодальной (Патриаршей) Библотеки 1: Рукописи греческие (Moscou, 1894), 85-87. Nous remercions la Section grecque de l'Institut de Recherche et d'Histoire des Textes d'avoir mis à notre disposition la traduction française inédite de Xénia Grichine (1961-1963) révisée par José Johannet (1995), cf. Aksinija Džurova, « La décoration des manuscrits grecs et slaves (IXe-XIe siècles), » Scripta 1 (2008) : (45-59) 52.

38 Cf. Staab, Pauluskatenen (voir note 4), 145-146; Robert Devreesse, Le fonds Coislin (vol. 2 de Catalogue des manuscrits grecs; Paris, 1945), 22-23.

39 Cf. Henri A. Omont, Inventaire sommaire des manuscrits grecs de la Bibliothèque Nationale et des autres bibliothèques de Paris et des départements 1 : Ancien fonds grec : théologie (Paris, 1886), 25; Staab, Pauluskatenen (voir note 4), 150-152. Si ce manuscrit a servi de modèle à l'édition reproduite par la PG 118-119, comme l'affirme Staab, cela signifie que l'éditeur a changé l'ordre des épîtres (voir note 9), harmonisé l'ordre des pièces précédant la chaîne (Argument d'Euthalius, liste des capitula, résumé court), qui est variable dans ce témoin, et même déplacé ceux des résumés courts qui étaient situés à l'intérieur de la chaîne.

40 Cf. Viktor E. Gardthausen, Catalogus codicum graecorum Sinaiticorum (Oxford, 1886), 56.

41 Cf. Enrico Stevenson, Codices manuscripti Palatini Graeci (Rome, 1885), 6 ; Staab, Pauluskatenen (voir note 4), 100-101; Enrica Follieri, «La minuscola libraria dei secoli IX e X, » dans La Paléographie grecque et byzantine: Actes du Colloque International du Centre Nationale de la Recherche Scientifique [21-25 octobre 1974] (éd. Jacques Bompaire, Jean Glénisson et Jean Irigoin ; Colloques internationaux du Centre National de la Recherche Scientifique 559; Paris, 1977), (139-164) 140. 42 Cf. Staab, Pauluskatenen (voir note 4), 127 ; Follieri, " La minuscola libraria dei secoli IX e $\mathrm{X} »$ (voir note 41), 140.

43 Cf. Staab, Pauluskatenen (voir note 4), 142-143; Elpidio Mioni, Codices graeci manuscripti Bibliothecae Divi Marci Venetiarum, Thesaurus antiquus 1: Codices 1-299 (Rome, 1981), 50-51. 
Marcianus gr. Z. 34 : Venise, Biblioteca Nazionale Marciana, gr. Z. 34 (coll. 349; Aland 1924), parch., XI ${ }^{\mathrm{e}}$ siècle, disposition marginale. ${ }^{44}$

Marcianus gr. Z. 546 : Venise, Biblioteca Nazionale Marciana, gr. Z. 546 (coll. 786, Aland 617), parch., $\mathrm{XI}^{\mathrm{e}}$ et XIII ${ }^{\mathrm{e}}$ siècle, disposition marginale. ${ }^{45}$

\subsection{Manuscrits contenant d’autres « résumés » de Jean Chrysostome}

Monacensis gr. 375: Munich, Bayerische Staatsbibliothek, gr. 375 (Aland 0142), parch., $\mathrm{X}^{\mathrm{e}}$ siècle, disposition à pleine page. ${ }^{46}$

Marcianus gr. Z. 35 : Venise, Biblioteca Nazionale Marciana, gr. Z. 35 (coll. 343, Aland 1925), parch., $\mathrm{xI}^{\mathrm{e}}$ siècle, disposition à pleine page. ${ }^{47}$

\section{Annexe 2 : « résumés courts » dans les manuscrits}

$\mathrm{E}=$ Argument d'Euthalius.

$\mathrm{T}$ = Argument attribué à Théodoret (résumé court).

al = autre Argument.

(c) = l’Argument apparaît comme un premier extrait de la chaîne.

$\emptyset=$ aucun Argument.

44 Staab, Pauluskatenen (voir note 4), 114-115; Mioni, Codices graeci 1 (voir note 43), 51-52. 45 Cf. Staab, Pauluskatenen (voir note 4), 144-145; Elpidio Mioni, Codices graeci manuscripti Bibliothecae Divi Marci Venetiarum, Thesaurus antiquus 2: Codices 300-625 (Rome, 1985), 441-443. 46 Cf. Ignaz Hardt, Catalogus codicum manuscriptorum Graecorum Bibliothecae Regiae Bavaricae 4: Cod. CCCXLVIII - CCCCLXXII continens (Munich, 1810), 108-119; Staab, Pauluskatenen (voir note 4), 160-161 («sekundärer Erweiterungs-Typus »).

47 Cf. Staab, Pauluskatenen (voir note 4), 162-163 (« sekundärer Erweiterungs-Typus »); Mioni, Codices graeci 1: Codices 1-299 (voir note 43), 52-53. 


\begin{tabular}{|c|c|c|c|c|}
\hline 䎹 & $\begin{array}{l}\underset{+}{+} \\
+\end{array}$ & $\begin{array}{l}\pi \\
+ \\
+\end{array}$ & Q & 山 \\
\hline 寻 & $\begin{array}{l}-4 \\
+ \\
4\end{array}$ & 되 & 디 & 되 \\
\hline 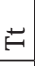 & $\begin{array}{l}4 \\
+ \\
-1\end{array}$ & 푀 & $\begin{array}{c}+ \\
+ \\
+4\end{array}$ & 더 \\
\hline$\stackrel{\Xi}{\sim}$ & $\begin{array}{c}\underset{+}{+} \\
\text { - } \\
\end{array}$ & 되 & 되 & 되 \\
\hline$\Xi$ & $Q$ & 되 & 디 & 떠 \\
\hline$\vec{E}$ & $\begin{array}{l}F \\
+ \\
+4\end{array}$ & & & w \\
\hline
\end{tabular}

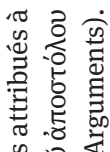

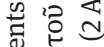

ฮิ कू⿻ स \& (ิ)

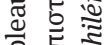

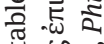

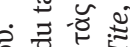
วิ

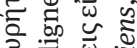
రి (1) ปี के है है ต่ ज : 0

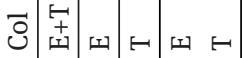

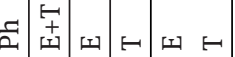

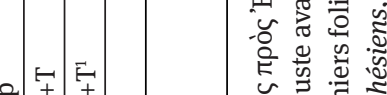

到南南Q四

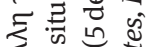
$\sum_{x \rightarrow 0}$ की

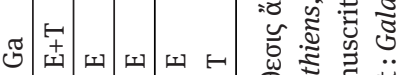
竎芯 ..

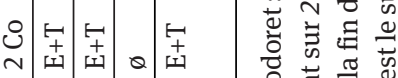

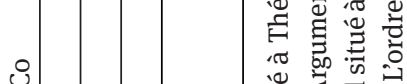

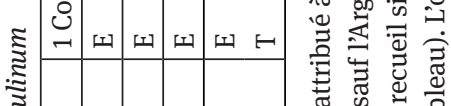
๘

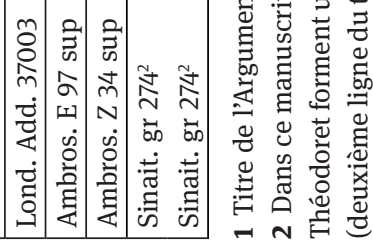

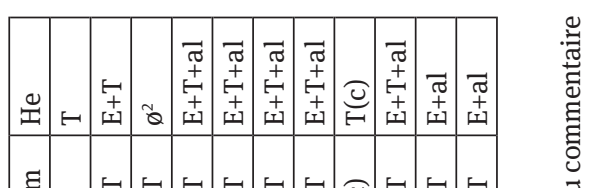

壳

귱

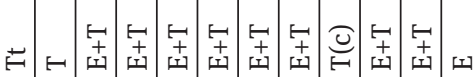

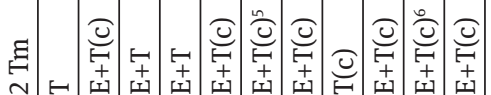

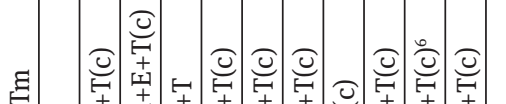

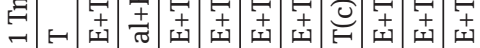

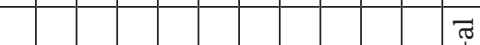

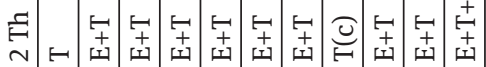

两

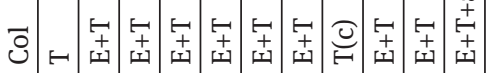

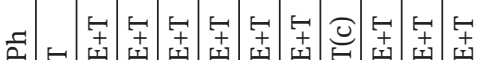

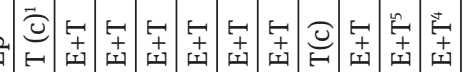

$: \overrightarrow{7}$

刃

志

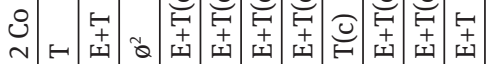

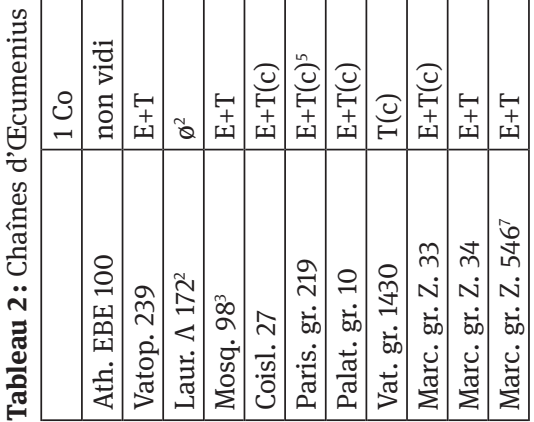

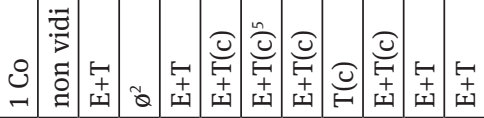

:

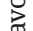

$\exists$

ᄋำ

प्य

$\stackrel{\infty}{\circ}$

ठั.

.

छ்

迹范

营 营密

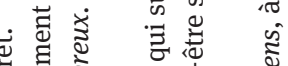

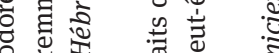

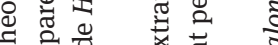

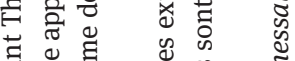

䒕范 过

芩

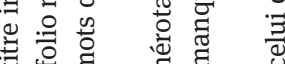

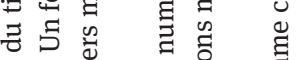

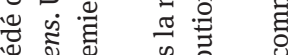

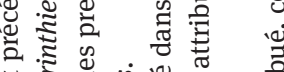

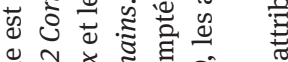

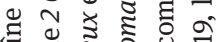

उै ปे की के है

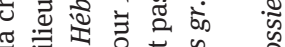

ข

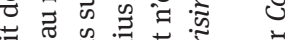

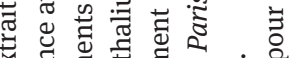

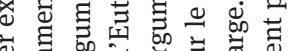

む)

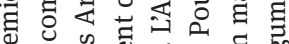

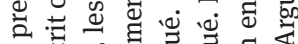

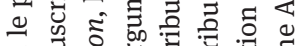

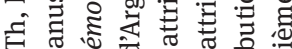

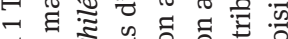

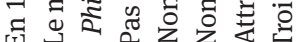




\section{Annexe 3 : Traduction des Arguments sur Philémon}

Jean Chrysostome

Philémon, un de ces hommes admirables et nobles - qu'il était admirable, c'est évident par le fait que sa maison entière était croyante . . . Donc cet homme admirable avait un serviteur, Onésime. Cet Onésime, donc, après avoir volé quelque chose à son maître, prit la fuite ... Arrivé chez Paul à Rome, l'ayant trouvé en prison, ayant bénéficié de son enseignement, il y reçut même le baptême.

Donc Paul écrit pour le recommander auprès de son maître, de sorte qu'il y ait pour tout une solution, et qu'il s'approche de lui comme désormais régénéré.

Mais comme certains affirment qu'il est superflu d'ajouter encore cette épître, puisqu'elle juge d'une affaire de peu d'importance, ... . vois donc combien de choses sont par là redressées.

La première, c'est d'être appliqué au sujet de tout ...

Deuxièmement, il ne faut pas désespérer de la race des esclaves . . . Car si le voleur, le fuyard est devenu si vertueux ..., a fortiori il ne faut pas désespérer d'hommes libres. résumé long

Donc Philémon était, dès

le début, un homme admirable, Phrygien de naissance, croyant, pour ce qui est de son mode de vie, prenant sa part aux besoins des saints et faisant des aumônes. Quant à Onésime, ce fut d'abord un fuyard (c'est que, après avoir volé de l'argent de la maison de Philémon, il prit la fuite) ; mais, venu à Rome, il écouta Paul et, après avoir été catéchisé par lui et baptisé, alors que Paul était en prison, il devint, lui aussi, quelqu'un d'admirable ... Il renvoie celui-ci [à Philémon], avec cette lettre aussi, en la faisant exhortative.

Certains disent qu'il ne faut pas compter celle-ci avec les autres, puisqu'elle est au sujet d'une affaire sans importance. Mais vois combien d'utilités en sont issues!

D’abord, elle nous apprend à nous appliquer même à l'égard de ce qui paraît sans importance...

Deuxièmement, que si un esclave si effronté et si méchant s'est converti, il ne faut pas désespérer de soi-même, surtout si on a été élevé dans la liberté. résumé court

Philémon était un homme admirable, Phrygien de naissance, croyant, pour ce qui est de son mode de vie et prenant sa part aux besoins des saints. Onésime, esclave de celui-ci, prit la fuite après l'avoir volé ; et, arrivé à Rome chez Paul, il fut catéchisé par lui et, après avoir été baptisé, alors que [Paul] était en prison, il devint admirable.

Il renvoie celui-ci à Philémon, en lui faisant cette lettre d'exhortation.

Certains disent qu'il ne faut pas la compter avec les autres épîtres, puisqu'elle est écrite au sujet d'une affaire sans importance. Mais vois combien d'utilités en sont issues!

D’abord, elle apprend à être appliqué même à l'égard de ce qui est sans importance.

Deuxièmement, que si un esclave si effronté s'est converti, il ne faut pas désespérer des gens libres. 


\section{Traduction}

Troisièmement, il ne convient pas d'arracher les esclaves à leurs maîtres. Car si Paul... . n’a pas consenti à retenir Onésime... contre l'avis de son maître, a fortiori, il ne faut pas que nous fassions cela. En effet, si le serviteur est admirable, c'est alors qu'il est le plus nécessaire qu'il reste dans la condition d'esclave...

Dirai-je autre chose? Il nous enseigne à ne pas rougir des serviteurs s'ils sont vertueux. Car si Paul ... dit tant de choses à son sujet, combien plus nous, au sujet des nôtres.

Donc, puisqu'il y a tant de vertus, alors même que nous n'avons pas encore tout dit, qui pense qu'il est superflu d'intégrer aussi cette épître?
Troisièmement, qu'il ne faut pas, sous prétexte de piété, arracher des esclaves à leurs maîtres si ceux-ci ne le veulent pas.

Quatrièmement, que nous n'avons pas à regarder de haut les esclaves s'ils sont vertueux, ni à rougir d'eux, puisque Paul appelle Onésime «enfant.»

Quel est donc celui qui dit qu'il ne vaut pas la peine de compter cette lettre avec les autres, alors qu'elle est remplie d'une si grande utilité?
Troisièmement, qu'il ne convient pas, sous prétexte de piété, arracher des esclaves contre le gré de leurs maîtres.
Quatrièmement, que nous n'avons pas à rougir des esclaves s'ils sont vertueux, puisque Paul appelle Onésime «enfant. »

Comment donc ne faudraitil pas compter avec les autres cette lettre qui a une si grande utilité? 


\section{Annexe 4 : Arguments sur Colossiens}

\begin{tabular}{|c|c|c|}
\hline Jean Chrysostome & résumé long & résumé court (texte intégral) \\
\hline 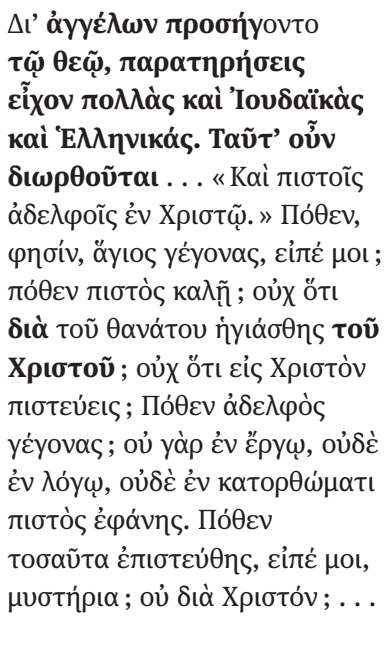 & & 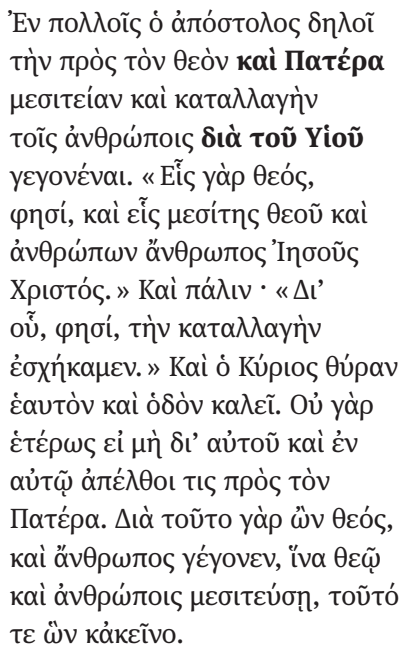 \\
\hline \multirow[t]{2}{*}{ 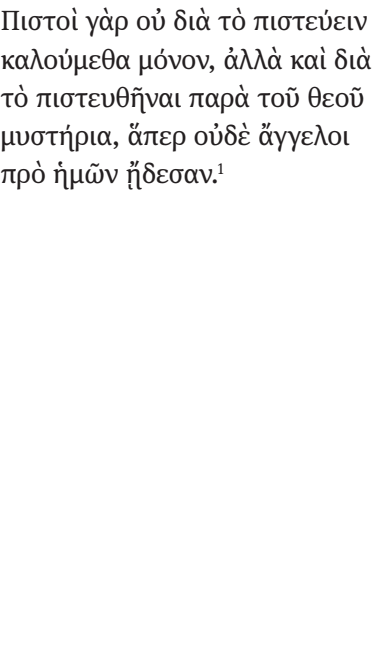 } & 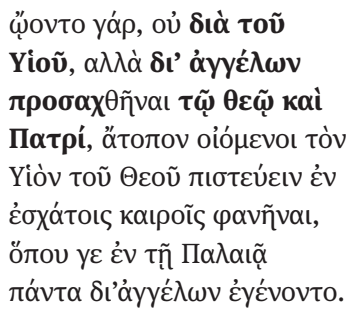 & 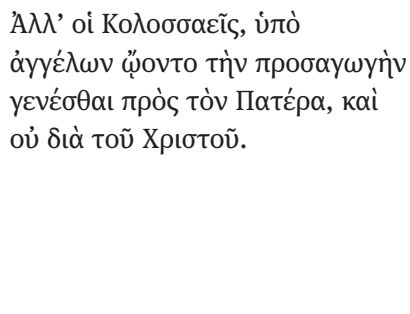 \\
\hline & 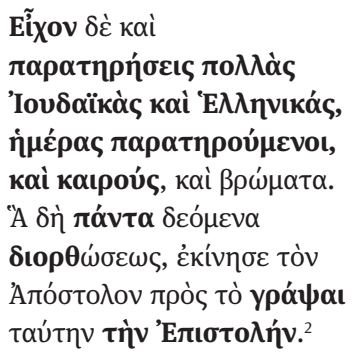 & 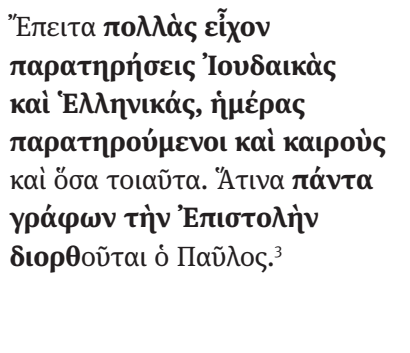 \\
\hline
\end{tabular}

1 Jean Chrysostome, In epistulam ad Colossenses commentarius, homilia 1,1-2 (PG 62:299-301).

2 Théophylacte de Bulgarie, Expositio Epistulae divi Pauli ad Colossenses (PG 124:1205).

3 Ps.-Théodoret, Argumentum Epistulae ad Colossenses (PG 119:12). 


\section{Traduction}

C'est par les anges qu'ils approchaient de Dieu, ils avaient de nombreuses observances judaïques et helléniques. C'est donc cela qu'il redresse ... « Et aux croyants, frères en Christ. » D’où, dit-il, es-tu devenu saint, dis-moi ? D’où t'appelles-tu croyant? N'est-ce pas parce que tu as été sanctifié par la mort du Christ? N'est-ce pas parce que tu crois au Christ? D'où es-tu devenu frère? Ce n'est pas par un acte, ni par une parole, ni par une œuvre de vertu que tu as été rendu croyant. D'où t'es-tu vu confier tous ces mystères? N'est-ce pas grâce au Christ?... Car ce n'est pas seulement à cause $\mathrm{du}$ fait de croire que nous nous appelons croyants, mais à cause du fait que nous soient confiés de la part de Dieu des mystères que des anges n'ont même pas connus avant nous.
Car ils estimaient que ce n'était pas par le Fils mais par des anges qu'ils s'étaient approchés du Dieu et Père, estimant qu'il était absurde de croire que le Fils de Dieu était apparu dans les derniers temps, là où, dans l'Ancien Alliance, tout était advenu par des anges.

Ils avaient aussi beaucoup d'observances judaïques et helléniques, observant les jours, les saisons et les aliments. Et comme, bien sûr, tout cela nécessitait un redressement, cela incita l'Apôtre à écrire cette épître.
À beaucoup d'endroits, l'Apôtre montre que la médiation et réconciliation à l'égard du Dieu et Père a eu lieu pour les hommes par l'intermédiaire du Fils. «Un seul Dieu, » dit-il, « et un seul médiateur entre Dieu et les hommes, un homme, Jésus-Christ. » Et encore : «Par lequel, » dit-il, «nous avons eu la réconciliation. » Et le Seigneur s'appelle lui-même «porte» et « voie.» Car ce n'est pas autrement que par lui et en lui que l'on se met en route vers le Père. Voilà pourquoi, alors qu'il est Dieu, il est aussi devenu homme, afin qu'il soit médiateur pour Dieu et les hommes, étant l'un et l'autre. Cependant, les Colossiens estimaient que c'était par des anges qu'advenait un rapprochement à l'égard du Père, et non par l'intermédiaire du Fils. Ensuite, ils avaient bien des observances judaïques et helléniques, observant jours, saisons et toutes choses de ce genre. C'est tout cela que Paul redresse en écrivant l'épître. 